\title{
L'éthique à l'écoute des neurosciences
}

\section{Jean Martin}

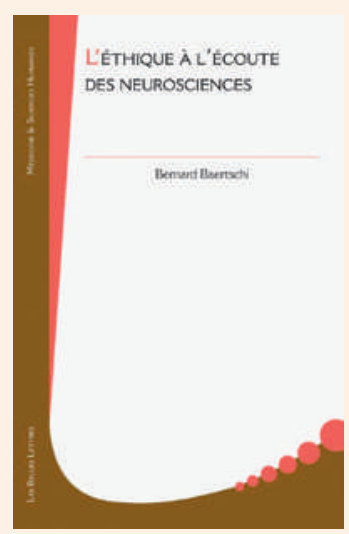

Bernard Baertschi L'éthique à l'écoute des neurosciences Paris: Edition Les Belles Lettres; 2013.

250 pages. 25 Euros. ISBN 978-2-251-43032-4
Bernard Baertschi est maître d'enseignement et de recherche à l'Université de Genève. Il est philosophe et très engagé sur des thèmes d'éthique, en rapport avec la dignité et l'intégrité de la personne ainsi que sur les questions suscitées par les développements de la biomédecine. Le présent ouvrage est centré, à propos des apports des neurosciences, sur l'éthique normative: son but est d'examiner les normes qui dirigent notre conduite, ainsi que les valeurs qui les inspirent, en les confrontant aux connaissances nouvelles.

Après une utile introduction, notamment pour le non-spécialiste, le premier chapitre rappelle les deux approches éthiques principales, déontologiste d'une part, conséquentialiste/utilitariste d'autre part. Est discuté le dilemme éthique devenu classique du wagon fou (trolley problem) - exemple traitant des possibilités d'intervention par une tierce personne, qui peuvent entraîner la mort soit d'une, soit de cinq personnes selon la décision prise. Le chapitre 2 est une présentation fouillée du principe des actions à double effet et des situations pratiques dans lesquelles il peut s'appliquer; enjeu dans beaucoup de débats bioéthiques, notamment en fin de vie. Discussion du choix des moyens et du moindre mal, de la place et du rôle de l'intention. S'agissant de la problématique de notre liberté d'action, entre les libertaires radicaux et les déterministes absolus/ durs (qui ne croient pas au libre arbitre des individus), Baertschi propose un modèle de déterminisme doux (soft), le compatibilisme. «Le déterminisme dur demande qu'on prive de toute signification morale la différence entre crime et maladie [...] Certains auteurs militant pour l'abandon du rétributivisme au niveau pénal [...] A mon sens, les neurosciences fournissent des données qui viennent à l'appui d'un déterminisme doux, contribuant par là à sauver la responsabilité et une certaine forme de libre arbitre».

Au chapitre 3 est abordée la question complexe de savoir si, au plan philosophique et au vu de certains critères, tous les êtres humains sont des personnes: sujet aussi très présent dans les défis aujourd'hui posés à l'éthique et aux soins médicaux ainsi que son corollaire de savoir si seuls des humains peuvent être des personnes... Autre interrogation lourde: «Existe-t-il des êtres humains incapables de moralité?» Thème qui préoccupe non seulement les éthiciens mais aussi la société, le système judicaire, les experts de la santé mentale, les psycho-sociologues. Sont traités le grand groupe des psychopathes, l'autisme, la maladie d'Alzheimer et autres dé- mences. «Les psychopathes ne sont peut-être pas des êtres humains à part entière, s'est demandé (le philosophe et médecin américain) Engelhardt et les expériences menées ne permettent pas vraiment de lever le doute [...]. Le doute est sérieux, car être une personne est la propriété qui fonde le statut moral des êtres humains.» Sont discutés les critères qui entrent en ligne de compte pour définir le statut de personne. Noter que, pour d'assez nombreux auteurs, ce qui constitue une personne est une propriété dont la possession - et la perte - est graduelle, et qu'il faudrait déterminer un seuil en deça duquel on cesse d'en être une.

\section{«Un ouvrage substantiel sur des problématiques très actuelles.»}

Puis, au chapitre 4, Baertschi présente cet aspect de la psychologie morale qu'est la réalité des tricheurs et des menteurs - et leur détection par diverses techniques quand les circonstances demandent de telles investigations. Le traitement détaillé de ces questions, y compris la prévention des comportements déviants, est suivi d'une réflexion sur l'«amélioration morale» - abordant le thème qui retient de plus en plus l'attention du enhancement (augmentation/rehaussement des capacités de l'être humain). Le titre de la conclusion interpelle: «La vérité importe-t-elle moralement?» (Avec «On doit admettre que, dans certains contextes, il existe des valeurs plus importantes que la vérité» - beau sujet.)

Un ouvrage substantiel sur des problématiques très actuelles, touchant plusieurs enjeux sociétaux qu'il n'est pas/plus possible de considérer de manière simpliste, en noir et blanc; ainsi la question du libre arbitre et celle de la responsabilité pénale ou civile. Même si les sujets sont complexes et si la lecture sera facilitée par des connaissances préalables de philosophie et psychologie, ce livre bien informé des recherches récentes se lit aisément. A recommander à celles et ceux que préoccupent les conséquences, morales notamment, des découvertes neuroscientifiques, susceptibles de modifier le regard porté sur des fondements même de nos cadres de référence, au plan médical, social et juridique en particulier. 